\title{
Improved fabrication efficiency of nanoporous alumina templates for nanostructure growt
}

\author{
Renan D. Domingos*, Fanny Béron
}

\begin{abstract}
A process to achieve alumina film cathodic delamination produced by hard anodization was developed during this work, opening the possibility to investigate the phenomena involved during the film delamination. The obtained free-standing films exhibits adequate structural and chemical characteristics. While the pore surface is being cleaned and polished during the cathodic etching process, it also ensures the formation of $\alpha-\mathrm{Al} 2 \mathrm{O} 3$, the most stable phase of aluminum hydroxide.
\end{abstract}

\section{Key words:}

Nanoporous anodic aluminum oxide, cathodic delamination, growth by metallic flux method.

\section{Introduction}

The effects of dimensionality reduction have attracted a lot of attention in the last decades due to quantum effects present in low dimensionality systems (nanowires, nanodots and antidots). Currently even more complex systems are the research focus in several laboratories in order to find novel technological solutions or still more complex phenomena. The synthesis and growth of these nanostructures depend on a great precision in their chemical composition, as well as dimensionality parameters of the nanostructures. New growth techniques are needed as well as the suitability of some parameters in the current processes, in order to obtain these materials. In this line of growth of new nanostructures we propose a new method for obtaining films of high purity alumina and optimized for nanostructure growth processes for the flux nanocrystallization method.

\section{Results and Discussion}

The growth of nanostructures by the flux nanocrystallization method ${ }^{[1]}$ uses high temperatures thus requiring that the template used does not chemically react with the other compounds present in the flux. Ceramics are known for their high heat resistance, thus a great choice to use as a template for growth, as well as in other techniques due to the chemical and physical stability of the alumina produced by this process.

The most frequent method used in the production of alumina films by hard anodization is by two stages of anodization using oxalic acid $(0.3 \mathrm{~mol})$ and a potential of $120 \mathrm{~V}^{[2]}$. During the first one, it occurs a pore organization in high purity aluminum (99.999\%). The oxide formed is then removed using an acidic solution composed of chromic and phosphoric acids, before performing a second anodization, using the same parameters than the first anodization. For some nanostructure growth techniques, acid solutions are still employed during the process to remove the aluminum remaining and to enlarge the alumina film pores.

The process described in this work uses the same oxalic acid solution at $0.3 \mathrm{~mol}$ to perform both the anodic and delamination processes of the alumina films. In addition to making it free of contaminants, the pores are attacked during the cathodic delamination process, thus obtaining a better physical appearance. After the process, it is possible to reuse the aluminum substrate for a next alumina film.
The cathodic delamination occurs when an inverse potential is applied on an anodizing cell. So far, this delamination potential is only reported for alumina produced by mild anodization. ${ }^{[3-5]}$ However, due to the low applied voltage in this case, it can not be directly transposed for hard anodization, since the chemical kinetics are different.

The process of cathodic delamination performed was inspired by the work of reference E.Choudhary et al. To determine the delamination potential for alumina produced at $120 \mathrm{~V}$, the current curves were monitored. No current increase was observed for potentials lower than $70 \mathrm{~V}$. However, it is responsible for the dissolution process of the less stable oxide formed during hard anodization, which is required for the quality of the desired alumina films. For applied potentials greater than -70V, the current increased, due to the formation of hydrogen gas at the interface between the aluminum oxide and substrate, which can be visually observed at the oxide surface. ${ }^{[4,5]}$ This gas is responsible for the film delamination, since the pressure it exerts at the interface, where the $\mathrm{H}^{+}$ions recombine with the electrons present in the electrochemical cell anode, allows the delamination to occur. For delamination, the gas formation at the interface must be greater than the permeability of the alumina and this excess is responsible for the delamination.

\section{Conclusions}

It was possible to obtain a delamination greater than $80 \%$ of the alumina film area, observing chemical and physical improvements in delaminated films. The process of delamination of films obtained by hard anodizing under other potentials follows a pattern in the delamination potential that we are still studying in order to improve the understanding of the process of gas formation and to measure the force necessary to delaminate this film through cathodic delamination process

\section{Acknowledgement}

I thank Professor Fanny Béron for guidance during this work and activities within the laboratory, CCS-Nano for the facilities and equipment made available for this work, as well as FAPESP for resources and equipment.

[1] INPI - BR 1020140197940

[2] W. Lee. Et al. Nature Materials, 2006, 5(9), 741-747.

[3] O. Nishinaga, T. et al., Scientific Reports, 2013, 3,1-6.

[4] E. Choudhary et al., RSC Advances, 2016, 6, 67992-67996.

[5] S. H. Jeong, et al., RSC Advances, 2017, 7, 4518-4530. 